\title{
Correction zu: Editorial PFG 5/2021
}

\section{Michael Cramer ${ }^{1} \cdot$ Wolfgang Kresse ${ }^{2}$}

Published online: 19 November 2021

(c) Deutsche Gesellschaft für Photogrammetrie, Fernerkundung und Geoinformation (DGPF) e.V. 2021

\section{Correction zu: PFG}

https://doi.org/10.1007/s41064-021-00180-x

Im Originalbeitrag war leider ein Fehler im zweiten Satz des zweiten Absatzes. Korrekt muss es lauten:

Ebenfalls vor genau 100 Jahren am 26. April 1921 entstand Heinrich Wilds Werkstatt für Feinmechanik und Optik in Heerbrugg.

Der Originalbeitrag wurde korrigiert.

Die Originalversion des Beitrags ist zu finden unter: https://doi. org/10.1007/s41064-021-00180-x.

Michael Cramer

michael.cramer@ifp.uni-stuttgart.de

$\triangle$ Wolfgang Kresse

kresse@hs-nb.de

1 Universität Stuttgart, Stuttgart, Germany

2 Hochschule Neubrandenburg, Neubrandenburg, Germany 